Ann. Biol. anim. Bioch. Biophys., I975, 15 (I), ro3-II4.

\title{
DIGESTIBILITÉ DES HUILES DE COLZA, DE CANBRA (') ET D'ARACHIDE. ÉTUDE DE LEUR LIPOLYSE IN VITRO ET IN VIVO
}

\author{
J. P. SERGIEL et G. ROCQUELIN \\ avec la collaboration technique de Lydia RECORBET \\ Station de Recherches sur la Qualité des Aliments de l'Homme, I. N. R. A., \\ 21034 Dijon Cedex
}

\section{RÉSUMÉ}

La digestibilité des huiles de colza, de canbra et d'arachide a été étudiée en comparant leur hydrolyse par la lipase pancréatique, d'une part in vitro et d'autre part, in vivo dans la lumière intestinale du Rat.

In vitro, la vitesse initiale d'attaque par la lipase pancréatique de l'huile de colza est inférieure à celle des huiles de canbra et d'arachide. Par contre, le taux d'hydrolyse à zo minutes est plus élevé pour l'huile de colza que pour les deux autres huiles.

In vivo, l'huile de colza provoque chez le Rat un ralentissement de l'évacuation gastrique du régime. Cependant, au cours de son transit intestinal, elle est aussi complètement hydrolysée par la lipase pancréatique que les deux autres huiles expérimentées. La moins bonne utilisation digestive de l'huile de colza n'est donc pas due à un déficit d'hydrolyse de ses triglycérides, mais à une mauvaise absorption de l'acide érucique sous forme libre. En effet, après $\mathrm{I} h 30$ et 3 heures de digestion, l'acide érucique représente respectivement $80 \mathrm{p}$. Ioo et $65 \mathrm{p}$. Ioo des acides gras totaux de la fraction "acides gras libres " de l'intestin grêle.

L'huile de colza (Brassica napus) renferme de fortes proportions d'acides gras monoènes à très longue chaîne qui sont l'acide $c i s-\Delta$-I I eicosénoïque $\left(\mathrm{C}_{20}:{ }_{1}\right)$ et l'acide cis- $\Delta-\mathrm{I}_{3}$ docosénoïque ou acide érucique $\left(\mathrm{C}_{22}: 1\right)$ qui représentent respectivement ro à I2 p. Ioo et 45 à $5^{\circ} \mathrm{p}$. Ioo des acides gras totaux. Ces acides gras ne sont présents qu'à l'état de traces dans l'organisme. Une autre particularité de cette huile est sa structure glycéridique : les acides gras $\mathrm{C}_{20: 1}$ et $\mathrm{C}_{22: 1}$ sont presque exclusivement estérifiés sur les positions externes des triglycérides qui les renferment (GRYNBERG et al., I966; MARCINKIEWICZ ot al., I 968$)$.

On sait que chez le Rat, l'utilisation digestive de l'huile de colya est inférieure à

(1) Cambra : Contratetions de Canadian brassica. 
celle d'autres huiles alimentaires (DEUEI et al., I948; ZrOMBSKI, I964 ; BEARE et al., I960). RocQueI.IN et al. (I969) ont comparé chez le Rat l'utilisation digestive des huiles de colza, de canbra (ne contenant que 0,5 à 2 p. Ioo d'acide érucique) et d'arachide, incorporées à des régimes à raison de $I_{5} \mathrm{p}$. Ioo en poids. Ils ont montré que le coefficient d'utilisation digestive apparent (CUD) de 1'huile de colza (8I p. Ioo) est inférieur à celui des huiles de canbra (95 p. 100) et d'arachide (92 p. I00). En outre, la méthode de fractionnement de ToulıEc et al. (I968), utilisée lors de l'extraction des lipides fécaux a permis de montrer que la fraction soluble dans le chloroforme-méthanol est deux fois plus importante que la fraction insoluble (constituée essentiellement par les acides gras des savons insolubles), ce qui est l'inverse des résultats obtenus avec l'huile d'arachide. L'acide érucique, principal responsable de l'utilisation digestive médiocre de l'huile de colza - puisque son CUD n'est que de $73 \mathrm{p}$. Ioo - est présent en fortes proportions aussi bien dans la fraction soluble que dans la fraction insoluble (79 et 76 p. roo des acides gras totaux respectivement).

Par ailleurs, RocQUELIN et al. (I97I) ont montré que l'huile de colza interestérifiée a une meilleure utilisation digestive que l'huile de colza normale ( $9 \mathrm{I}$ p. Ioo et 8 I p. Ioo respectivement). L'interestérification, en répartissant au hasard 1'acide érucique sur les 3 positions du glycérol ramène une partie des chaînes éruciques sur la position 2. Le CUD de l'acide érucique de l'huile de colza interestérifiée ( 85 p. roo) est plus élevé que celui de l'huile de colza normale (73 p. IOO). Ceci donne à penser que l'acide érucique sous forme libre serait moins bien absorbé que sous forme de 2 monoérucine.

Différentes hypothèses peuvent expliquer la médiocre utilisation digestive de l'huile de colza et la répartition quantitative particulière des lipides fécautu entre les fractions soluble et insoluble chez les animaux ayant ingéré cette huile :

- Ou bien la présence des acides gras à très longue chaîne, répartis de façon particulière dans les triglycérides de l'huile de colza modifie l'activité de la lipase pancréatique, dont la spécificité vis-à-vis des positions I et 3 des triglycérides est bien connue. Ceci entraînerait alors la formation de glycérides partiels riches en acides eicosénoïque et érucique ou de triglycérides intacts qui seraient éliminés en grande quantité dans la fraction soluble des lipides fécaux.

- Ou bien l'activité de la lipase n'est pas modifiée et il y a libération équivalente de tous les acides gras à partir des triglycérides de 1'huile. L'acide érucique libéré serait alors mal absorbé pour des raisons qui lui sont propres et qui restent à déterminer. Comme on sait qu'il ne forme pas préférentiellement de savons insolubles, il serait alors excrété en forte quantité sous forme libre, dans la fraction soluble des lipides fécaux.

Nous avons tenté de déterminer la valeur de ces hypothèses en comparant 1'activité de la lipase pancréatique vis-à-vis des huiles de colza, de canbra et d'arachide. Pour cela, nous avons utilisé 2 voies d'approche :

- étude in vitro de la vitesse initiale de l'hydrolyse, par la lipase pancréatique, d'huiles de colza et de canbra normales et interestérifiées afin de dissocier partiellement les facteurs " composition en acides gras " et " structure glycéridique ". Le substrat de référence est l'huile d'arachide;

- étude in vivo des proportions des différentes classes de glycérides des contenus digestifs de rats ayant ingéré des régimes renfermant soit de l'huile de colza, soit de l'huile de canbra, soit de l'huile d'arachide. 


\section{MATÉRIEL, ET MÉTHODES}

\section{1. .... Hydrolyse enzymatique in vitro}

Les déterminations des vitesses initiales d'hydrolyse par la lipase pancréatique ont été effectuées sur des huiles de colza et de canbra normales et interestérifiées et sur une huile d'arachide. Toutes ces huiles étaient raffinées. La composition du milieu d'hydrolyse est la suivante :

- $5 \mathrm{~g}$ d'une émulsion à Io p. Ioo d'huile dans une solution de gomme arabicue à to p. Ioo,

- I $\mathrm{ml}$ d'une solution de taurocholate de sodium à $20 \mathrm{p}$. IoO,

- $8 \mathrm{ml}$ d'une solution de $\mathrm{CaCl}_{2} \mathrm{O}, \mathrm{O} 2 \mathrm{M}$,

- I ml d'une solution de $\mathrm{NaCl} \mathrm{M}$,

- $0,5 \mathrm{ml}$ d'une solution de lipase à $20 \mathrm{mg} / \mathrm{ml}$ dans un tampon à $\mathrm{pH} 8,2$,

- eau distillée pour obtenir un volume final de $30 \mathrm{ml}$.

Ce milieu réactionnel sans lipase est agité et porté à $37^{\circ} \mathrm{C}$. Le pH est amené à 8,2 par addition de soude $0,3 \mathrm{~N}$. On ajoute alors la lipase et on enregistre pendant 30 minutes la courbe de neutralisation des acides gras libérés à l'aide d'un pHmètre titrimètre enregistreur Radiometer. A partir de cette courbe on détermine, d'une part, la vitesse initiale d'hydrolyse lipasique définie par la pente de la tangente à la courbe à I minute d'hydrolyse, et d'autre part, le degré d'hydrolyse à 30 minutes qui est la proportion d'acides gras libérés à partir des acides gras totaux des triglycérides d'origine.

\section{II. - Étude in vivo sur le Rat}

Des rats mâles pesant 230 à $250 \mathrm{~g}$, sont répartis en 3 lots de 6 rats et mis en cages individuelles. Ils sont alors conditionnés pendant I semaine à ingérer une quantité restreinte mais connue de régime test dans les délais les plus courts. Pour cela, ils sont mis à jeun pendant 16 heures avant de recevoir environ $3 \mathrm{~g}$ de régime expérimental (matière sèche) contenant I $5 \mathrm{p}$. Ioo en poids d'huile. Une fois le régime ingéré, on leur donne, ad libitum, pendant 8 heures de la provende commerciale et on recommence l'opération le jour suivant. Après une semaine de ce traitement quotidien, les rats sont capables d'ingérer la quantité de régime test dans un délai inférieur à 3 minutes. Nous avons préféré cette méthode à celle de l'intubation gastrique car elle offre l'avantage de présenter les huiles étudiées dans un régime complet, équilibré et homogène, identique à celui que nous avons adopté jusqu'à présent dans tous nos protocoles. La composition exacte de ce régime est rapportée dans le travail de Rocquelin et Leclerc (I969).

La quantité de régime consommée est déterminée par pesée, ce qui permet de connaître la quantité exacte de lipides ingérés. Les rats sont sacrifiés par décapitation I $h$ 30 ou 3 heures après la prise de nourriture ( 3 rats par lot dans chaque cas).

L'estomac et l'intestin grêle subdivisé en duodénum et partie distale jusqu'au cæcum sont ligaturés, puis rapidement prélevés séparément. Le contenu de chacun de ces segments est entraîné à l'aide d'une solution saline à 7,3 p. I ooo. Les lipides de ces contenus sont extraits selon la méthode de Folch et al. (I957) en tenant compte, pour le lavage de l'extrait lipidique, du volume de la solution saline utilisée lors du rinçage. La quantité d'extrait lipidique total est déterminée par pesée.

\section{III. - Techniques d'analyse chromatographique}

Les différentes classes lipidiques constitutives des contenus digestifs (mono, di, triglycérides et acides gras libres) sont séparées par chromatographie sur couches minces préparatives de gel de silice et évaluées quantitativement après récupération, par chromatographie en phaze gazeuse des esters méthyliques des acides gras par référence à un étalon interne (heptadécanoate de méthyle).

La chromatographie sur couches minces est effectuée sur des plaques de $20 \mathrm{~cm} \times 20 \mathrm{~cm}$ préparées selon STahL (I956). L'épaisseur de la couche de gel de silice est de $500 \mu$ et avant l'utilisation des plaques on effectue un "lavage" par migration dans un solvant constitué de chloroforme-méthanol-acide acétique $80: 25:$ I $\mathrm{v} / \mathrm{v} / \mathrm{v}$. La quantité de lipides appliquée sur chaque plaque est de l'ordre de I $5 \mathrm{mg}$. La séparation des différentes classes glycéridiques est effectuée par migration dans le milieu préconisé par Brown et al. (1962), constitué de : hexane, éther éthylique, méthanol, acide acétique $90: 20: 3: 2 \mathrm{v} / \mathrm{v} / \mathrm{v} / \mathrm{v}$. Les différentes bandes sont localisées par visualisation sous lumière ultra violette après pulvérisation d'une solution éthanolique à o, 2 p. Ioo 
de $2^{\prime} 7^{\prime}$ dichlorofluorescéine, et identifiées par comparaison avec des échantillons connus déposés simultanément sur la plaque. Chacune des bandes est alors récupérée par grattage et les lipides sont élués du gel de silice par de l'éther éthylique ou du chloroforme-méthanol pour être analysés par chromatographie en phase gazeuse.

La chromatographie en phase gazeuse des esters méthyliques est effectuée en isotherme sur des appareils équipés de détecteurs à ionisation de flamme d'hydrogène. Nous utilisons des colonnes en acier inoxydable de $3 \mathrm{~m}$ de long et de $2,2 \mathrm{~mm}$ de diamètre intérieur, remplies de chromosorb AW HMDS 6o-80 mesh imprégné de so p. Ioo de phase polaire BDS (butanediol succinate).

Les esters méthyliques des acides gras sont préparés selon MuNsch (1970) par transméthylation à chaud dans un mélange ternaire méthanol-benzène-acide sulfurique 1oo: $50: 10, \mathrm{v} / \mathrm{v} / \mathrm{v}$ et extraits à l'hexane.

Les acides gras sont identifiés par référence à des échantillons de composition connue et la valeur de chaque pic est calculée en faisant le produit de sa hauteur par sa distance de rétention.

\section{RÉSULTATS ET DISCUSSION}

\section{I. - Hydrolyse enzymatique in vitro}

Les résultats obtenus sur 30 essais réalisés avec chacune des huiles sont rapportés dans le tableau I. L'analyse statistique des valeurs concernant la vitesse initiale montre que les différences sont significatives entre les huiles d'arachide et de colza, ainsi que entre les huiles d'arachide et de canbra pour $\mathrm{P}<0, \mathrm{or}$. Si on donne à la valeur obtenue pour l'huile d'arachide considérée comme témoin l'indice Ioo, on aura 90 pour le colza et I Io pour le canbra. Il semble donc qu'au niveau de la vitesse initiale, c'est-à-dire au cours des premières minutes d'hydrolyse, il y ait un léger ralentissement de l'attaque lipasique de l'huile de colza. On pourrait penser que ce phénomène est dû à la présence presque exclusive des acides érucique et eicosénoïque en position externe dans les triglycérides de l'huile de colza. Néanmoins, nos essais effectués sur 1'huile de colza interestérifiée n'ont pas montré de différence de la vitesse initiale par rapport à l'huile de colza normale. Cette observation donne à penser que la structure particulière de 1'huile de colza n'affecte par directement sa vitesse initiale d'attaque par la lipase pancréatique. I a différence observée par rapport à l'huile d'arachide viendrait de la forte teneur en acide érucique mais ce point demande à être vérifié. Ce résultat est à rapprocher de ceux obtenus par BotTINo et al. (I967) qui observent une résistance à 1'hydrolyse par la lipase pancréatique, de triglycérides d'huiles provenant d'animaux marins et contenant sur les positions I et 3 des acides gras polyinsaturés à très longue chaîne comme le $\mathrm{C}_{20: 5}$ et le $\mathrm{C}_{22}:{ }_{8}$.

La différence observée entre l'huile d'arachide et l'huile de canbra en ce qui concerne la vitesse initiale est plus difficile à expliquer dans l'état actuel des connaissances. En effet, ces deux huiles ont une structure glycéridique voisine (SERGIEL I973) et le seul facteur que 1'on pourrait invoquer réside dans la différence des proportions en acides gras saturés totaux de ces deux huiles $(6 \mathrm{p}$. Ioo pour le canbra et $20 \mathrm{p}$. Ioo pour l'arachide). Toutefois, cette hypothèse mérite d'être vérifiée car on sait par ailleurs que la lipase ne semble pas avoir de spécificité pour les chaînes de I2 à I8 atomes de carbone (SAVARY et al., I956 ; MATTSON et al., I956), qu'elles soient saturées ou insaturées.

Le taux d'hydrolyse après 30 minutes des différentes huiles étudiées (tabl. I) ne présente pas un parallélisme absolu avec les résultats obtenus pour les vitesses initiales. L'huile de colza a un taux d'hydrolyse supérieur aux huiles de canbra et 
d'arachide et cette différence est significative pour $\mathrm{P}<0,0 \mathbf{I}$. D'autre part, l'interestérification de l'huile de colza abaisse son taux d'hydrolyse au niveau de celui de l'huile d'arachide alors qu'il n'y a pas de variation significative pour l'huile de canbra interestérifiée par rapport à 1 'huile normale.

\section{TABLEAU I}

Vitesse initiale de l'hydrolyse des différentes huiles étudiées (en $\mathrm{ml}$ de soude $0,3 \mathrm{~N}$ délivrés par unité de temps) et degré d'hydrolyse après 30 minutes

(quantité d'acides gras libérés pour Ioo acides gras des triglycérides d'origine)

\begin{tabular}{|c|c|c|c|c|c|}
\hline & \multicolumn{5}{|c|}{ Huiles } \\
\hline & Huile de colza & $\begin{array}{l}\text { Huile de colza } \\
\text { interestérifiée }\end{array}$ & $\begin{array}{c}\text { Huile } \\
\text { d'arachide }\end{array}$ & Huile de canbra & $\begin{array}{l}\text { Huile de canbra } \\
\text { interestérifiée }\end{array}$ \\
\hline Vitesse initiale & $0,215 \pm 0,009^{*}$ & $0,213 \pm 0,009^{*}$ & $0,238 \pm 0,003$ & $0,263 \pm 0,004^{*}$ & $0,258 \pm 0,005^{*}$ \\
\hline $\begin{array}{l}\text { Taux d'hydro- } \\
\text { lyse à } 30 \mathrm{mn}(\%)\end{array}$ & $43,4 \pm 0,6^{*}$ & $39,6 \pm 0,6$ & $39,2 \pm 0,6$ & $41,8 \pm 0,8$ & $13,6 \pm 1,1^{*}$ \\
\hline
\end{tabular}

* : Différence significative par rapport à l'huile d'arachide pour $\mathrm{P}<0,01$.

II. - Étude de l'hydrolyse in vivo des huiles de colza, de canbra et d'arachide

a) Les résultats concernant les quantités de lipides ingérées et les proportions par rapport aux ingérés de lipides totaux retrouvés dans l'estomac, le duodénum et la partie distale de l'intestin grêle I h 30 et 3 heures après l'ingestion des huiles sont rassemblés dans les tableaux 2 et 3 .

TABLEAU 2

Quantités de lipides ingérées (en $\mathrm{mg}$ )

par les rats sacrifiés 1 heure 30 et 3 heures après la prise de nourviture

(moyennes et valeurs extrêmes)

\begin{tabular}{c|c|c|c}
\hline & $\begin{array}{c}\text { Huile } \\
\text { d'arachide }\end{array}$ & $\begin{array}{c}\text { Huile } \\
\text { de colza }\end{array}$ & $\begin{array}{c}\text { Huile } \\
\text { de canbra }\end{array}$ \\
$\begin{array}{c}\text { Lipides ingérés par les rats } \\
\text { sacrifiés après 1 h 30 }\end{array}$ & $\begin{array}{c}491 \\
(442-519)\end{array}$ & $\begin{array}{c}4 \text { tix } \\
(155-475)\end{array}$ & $\begin{array}{c}459 \\
(140-481) \\
\text { lipides ingérés par les rats } \\
\text { sacrifiés après 3 heures }\end{array}$ \\
\end{tabular}


Dans l'estomac, la proportion de lipides retrouvés est légèrement supérieure dans le lot colza par comparaison aux lots arachide et canbra. Cette constatation nous donne à penser qu'il y a, malgré les variations individuelles, un retard dans l'évacuation gastrique du régime contenant de l'huile de colza, par rapport aux deux autres régimes. Ceci mériterait d'être confirmé sur un nombre plus élevé de rats. Ce résultat explique en partie celui de Thomasson (1956) qui observe que le temps de demi-disparition de la matière grasse ingérée, à partir du tractus gastro-intestinal entier de rat, est de 5 à 6 heures pour des corps gras bien absorbés comme le beurre et 1'huile de soja, et de 9 heures pour l'huile de colza. D'autre part, ZIOMBSKI (Ig62) qui a étudié la courbe de lipémie chez le rat nourri avec différentes graisses alimentaires (beurre, lard, huile d'olive, coprah, huile de colza) a trouvé un maximum retardé dans le temps avec l'huile de colza par rapport aux autres matières grasses.

Dans l'intestin (duodénum et partie distale de l'intestin grêle) le transit digestif semble s'accomplir de la même façon dans les 3 lots. En effet, le rapport entre la quantité de lipides retrouvés dans le segment et la quantité totale de lipides récupérés dans l'ensemble du tractus gastro-intestinal étudié est constant en fonction du temps dans tous les lots (tabl. 4).

\section{TABLEAU 3}

Proportions de lipides totaux vetrouvés (p. Ioo par rapport à la quantité ingérée) dans l'estomac, le duodénum et la partie distale de l'intestin grêle, 1 h 30 et 3 heures après ingestion d'huile d'arachide, de colza ou de canbra (moyennes et valeurs extrêmes)

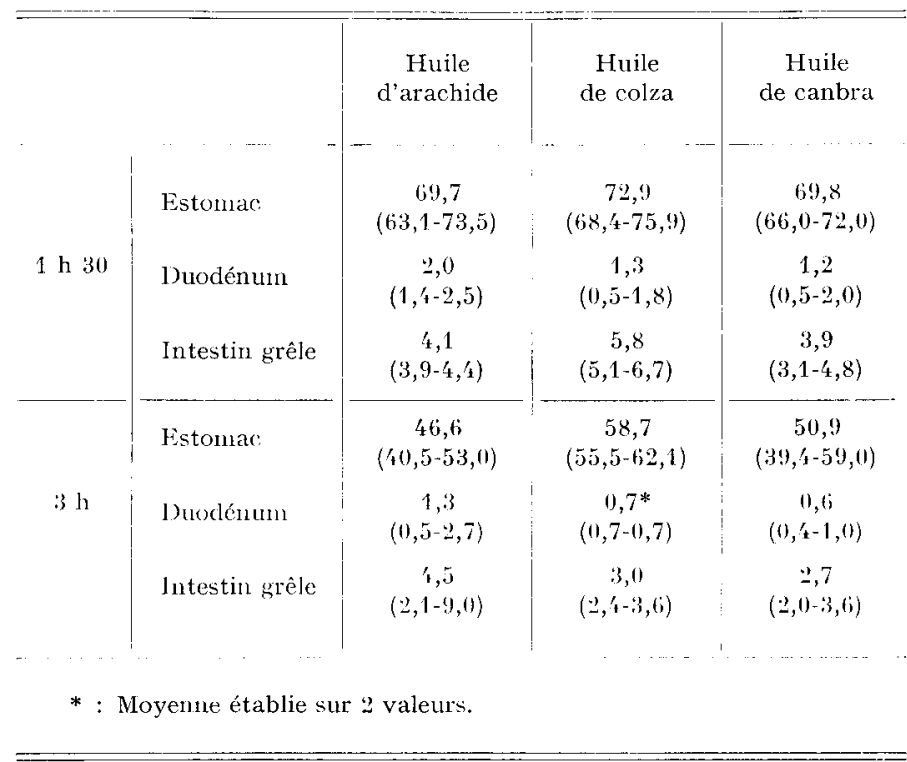

b) Les résultats concernant la répartition en mono, di, triglycérides et acides gras libres des différents contenus digestifs sont rassemblés dans le tableau 5 . 


\section{TABLEAU 4}

Proportions (p. 1oo) des lipides présents dans l'estomac, le duodénum et la partie distale de l'intestin grêle par rapport à l'ensemble des lipides retrouvés dans ces 3 segments du tractus digestif

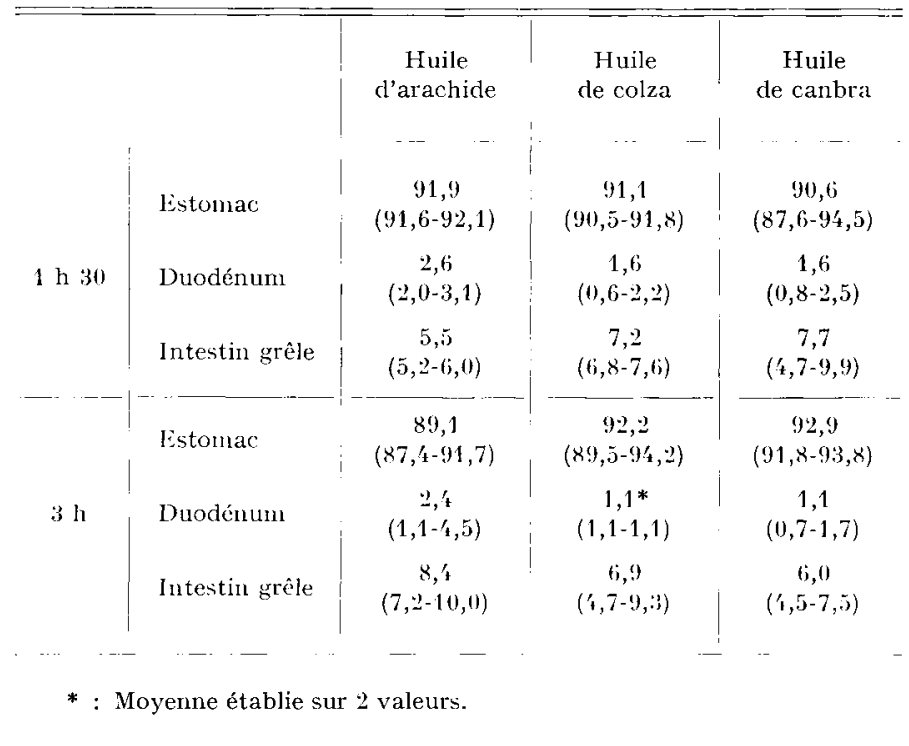

- Au niveau de l'estomac, 1'hydrolyse des triglycérides du régime est loin d'être négligeable et elle engendre surtout des acides gras libres et des diglycérides. On peut penser que le reflux de la lipase pancréatique vers l'estomac au cours du jet̂ne de $\mathbf{r} 6$ heures pourrait contribuer à cette hydrolyse relativement importante des triglycérides à chaîne longue de nos huiles. Cependant, cette hydrolyse des triglycérides par l'estomac a déjà été signalée par CLARK et al. (I969) qui trouvent in vitro une action lipolytique avec des homogénats de muqueuse gastrique de rats après exclusion de toute contamination pancréatique par dérivation prolongée de la sécrétion pancréatique. L'activité de la lipase gastrique serait assez forte vis-à-vis des triglycérides à chaîne courte et moyenne, et plus réduite vis-à-vis des triglycérides renfermant des chaînes de l'ordre de 18 atomes de carbone et plus. Enfin, un travail plus récent de Hamosh et al. (I973) fait état d'une activité lipasique qui serait présente dans les glandes séreuses de la langue de Rat et qui catalyserait la digestion des triglycérides en glycérides partiels et en acides gras libres. Son $\mathrm{pH}$ d'activité se situerait à $4,5-5,4$ c'est-à-dire à une valeur voisine de celle de l'estomac.

Il n'est donc pas invraisemblable dans nos conditions expérimentales, que l'hydrolyse stomacale des triglycérides résulte de l'action conjuguée de ces 3 lipases.

- Au niveau du duodénum qui est un site d'activité intense de la lipase pancréatique, c'est-à-dire d'hydrolyse des triglycérides, il semblerait, malgré les fortes variations individuelles, qu'après I h 30 , la proportion de triglycérides soit légèrement plus élevée dans le lot colza que dans les deux autres lots. Cette tendance générale se maintiendrait après 3 heures. A ce niveau de l'intestin, nos résultats traduiraient 


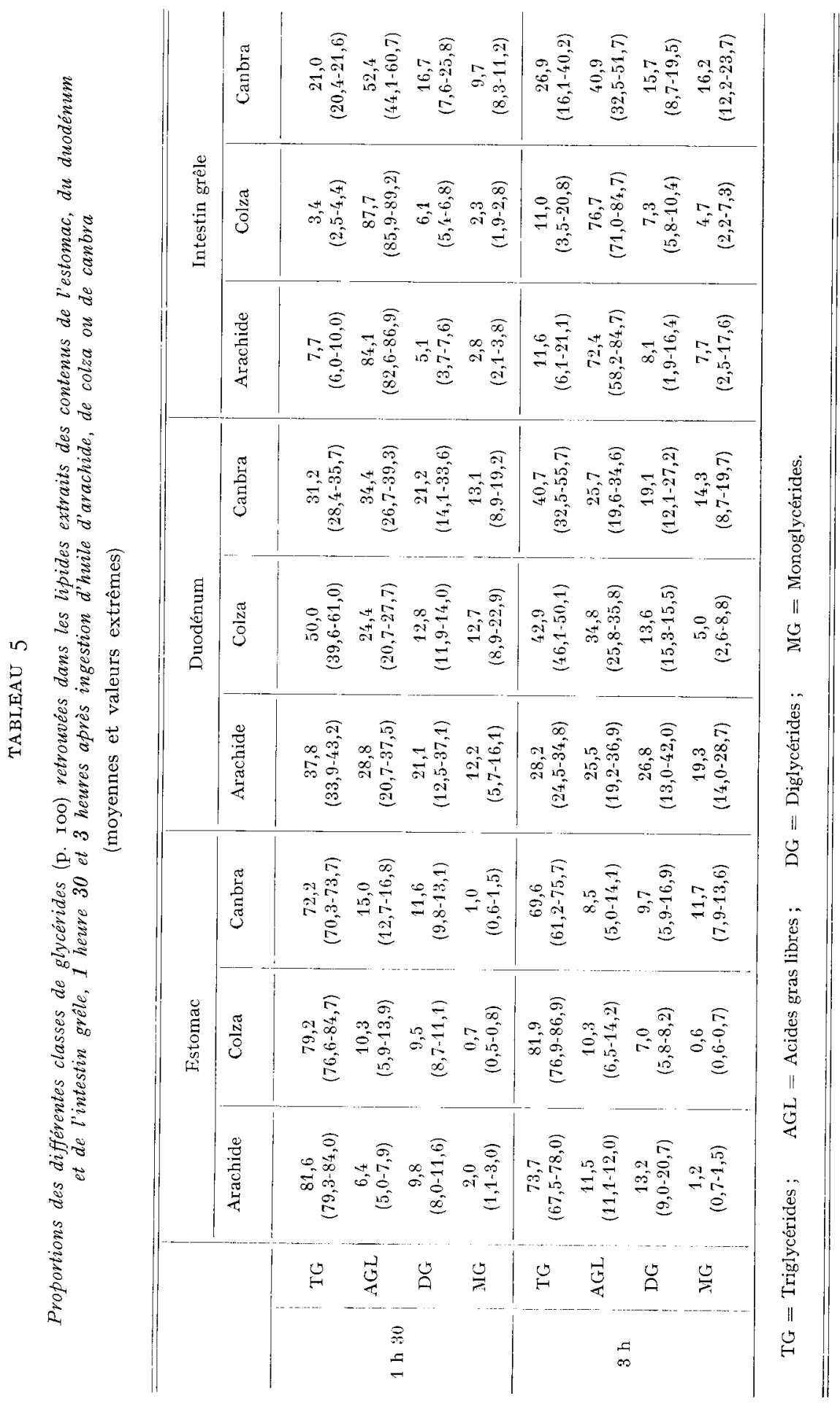


donc un ralentissement de la vitesse d'hydrolyse des triglycérides de l'huile de colza et seraient à rapprocher de ceux obtenus pour les vitesses initiales d'hydrolyse in vitro. D'autre part, ce phénomène pourrait éventuellement expliquer le retard de l'évacuation gastrique observé chez les rats ingérant le régime contenant l'huile de colza.

- Dans la partie distale de l'intestin grêle qui est à la fois un lieu de digestion et surtout d'absorption des lipides, notamment au niveau du jejunum, on ne trouve que très peu de triglycérides chez les sujets ayant ingéré des huiles de colza et d'arachide. La classe qui domine largement, aussi bien après I heure 30 qu'après 3 heures est celle des acides gras libres qui représentent 72 à $87 \mathrm{p}$. Ioo des lipides totaux récupérés dans ce segment intestinal. Par ailleurs, l'analyse des acides gras des différentes classes du contenu de l'intestin grêle du lot colza (tabl. 6) montre que dans la frac-

\section{TABLEAU 6}

Proportions (en p. Ioo des acides gras totaux) de $C_{20}: 1$ et de $C_{22}: 1$ dans les différentes classes lipidiques des contenus du duodénum ct de la partie distale de l'intestin grêle 1 heure 30 et 3 heures après ingestion du régime contenant de l'huile de colza

(moyennes et valeurs extrêmes)

\begin{tabular}{|c|c|c|c|c|c|c|}
\hline & & & $\mathrm{TG}$ & $\mathrm{AGL}$ & $D G$ & $\mathrm{MG}$ \\
\hline \multirow{4}{*}{ Duodénum } & \multirow{2}{*}{ 1 h 30} & $C_{20: 1}$ & $\begin{array}{c}5,2 \\
(3,9-6,2)\end{array}$ & $\begin{array}{c}5,9 \\
(5,3-6,7)\end{array}$ & $\begin{array}{c}3,9 \\
(2,6-4,7)\end{array}$ & $\begin{array}{c}1,5 \\
(1,1-1,8)\end{array}$ \\
\hline & & $C_{22}: 1$ & $\begin{array}{c}30,0 \\
(19,8-37,5)\end{array}$ & $\begin{array}{c}34,4 \\
(28,6-40,0)\end{array}$ & $\begin{array}{c}19,0 \\
(14,1-27,2)\end{array}$ & $\begin{array}{c}3,7 \\
(2,5-4,6)\end{array}$ \\
\hline & \multirow{2}{*}{$3 \mathrm{~h}$} & $C_{20: 1}$ & $\begin{array}{c}4,7 \\
(4,1-5,3)\end{array}$ & $\begin{array}{c}4,5 \\
(3,8-5,2)\end{array}$ & $\begin{array}{c}2,0 \\
(1,8-2,3)\end{array}$ & traces \\
\hline & & $\mathrm{C}_{22: 1}$ & $\begin{array}{c}24,9 \\
(19,0-30,8)\end{array}$ & $\begin{array}{c}26,2 \\
(22,9-29,5)\end{array}$ & $\begin{array}{c}9,4 \\
(6,8-12,1)\end{array}$ & $\begin{array}{c}1, \mathbf{4} \\
(1,1-1,7)\end{array}$ \\
\hline \multirow{4}{*}{$\begin{array}{l}\text { Intestin } \\
\text { grêle }\end{array}$} & \multirow{2}{*}{$1 \mathrm{~h} 30$} & $C_{20}: 1$ & $\begin{array}{c}2,6 \\
(1,2-3,9)\end{array}$ & $\begin{array}{c}4,9 \\
(3,1-6,4)\end{array}$ & $\begin{array}{c}2,0 \\
(1,4-2,4)\end{array}$ & $\begin{array}{c}3,4 \\
\left(2,2-4,4_{t}\right)\end{array}$ \\
\hline & & $C_{22: 1}$ & $\begin{array}{c}13,4 \\
(6,6-236)\end{array}$ & $\begin{array}{c}80,3 \\
(77,3-82,2)\end{array}$ & $\begin{array}{c}12,2 \\
(10,0-16,3)\end{array}$ & $\begin{array}{c}22,6 \\
(15,8-33,1)\end{array}$ \\
\hline & \multirow{2}{*}{$3 \mathrm{~h}$} & $\mathrm{C}_{20: 1}$ & $\begin{array}{c}3,0 \\
(2,6-3,2)\end{array}$ & $\begin{array}{c}6,1 \\
(5,6-6,5)\end{array}$ & $\begin{array}{c}3,9 \\
(3,7-1,2)\end{array}$ & $\begin{array}{c}2,5 \\
(1,9-3,1)\end{array}$ \\
\hline & & $C_{22}: 1$ & $\begin{array}{c}12,6 \\
(9,4-16,8)\end{array}$ & $\begin{array}{c}65,4 \\
(59,6-73,8)\end{array}$ & $\begin{array}{c}19,8 \\
(14,3-26,4)\end{array}$ & $\begin{array}{c}16,2 \\
(13,7-18,5)\end{array}$ \\
\hline
\end{tabular}

tion «acides gras libres ", 1'acide érucique représente 77 à 82 p. Ioo des acides gras totaux après I heure 30 et 60 à 74 p. Ioo après 3 heures. Il apparaît donc que l'hydrolyse est aussi complète pour les triglycérides de l'huile de colza que pour ceux de l'huile d'arachide, et que 1'acide érucique sous forme libre est présent en forte proportion dans la lumière intestinale. Ces résultats sont à rapprocher de ceux de SAvary et al. (I966) qui ont observé que la triérucine est hydrolysée de façon presque totale pendant le transit intestinal. Nous avons nous-même confirmé ce point lors d'une étude 
récente sur les formes d'élimination fécale de l'acide érucique chez des rats ayantingéré de l'huile de colza (à paraître). L'analyse de la fraction soluble montre que les acides gras libres représentent $95 \mathrm{p}$. Ioo des lipides totaux de cette fraction, le reste étant réparti entre les diglycérides et les triglycérides.

Les raisons de la mauvaise utilisation digestive de l'acide érucique sous forme libre n'apparaissent donc pas très clairement si on le compare à d'autres acides gras mal absorbés comme les acides saturés palmitique et stéarique. En effet, il ne forme pas préférentiellement de savons insolubles comme ces derniers. Par ailleurs, son point de fusion est de $33^{\circ} \mathrm{C}$, et sa solubilisation micellaire à $37^{\circ} \mathrm{C}$ - condition nécessaire à l'absorption des lipides — n'est pas négligeable. D'après SAvary et al. (Ig66) elle est de $I 5$ mmoles/I pour l'acide oléique, I, 5 mmoles/I pour l'acide palmitique et $8 \mathrm{mmoles} / \mathrm{I}$ pour l'acide érucique.

En conclusion, les résultats que nous avons obtenus in vitro et in vivo sur l'hydrolyse par la lipase pancréatique de l'huile de colza permettent de répondre à l'une des questions que nous nous sommes posées à l'origine de ce travail.

Les expériences in vitro ont permis de constater que la structure glycéridique particulière de l'huile de colza n'a pas d'effet marqué sur le degré d'hydrolyse par la lipase pancréatique après 30 minutes. D'autre part, les différences observées en ce qui concerne la vitesse initiale paraissent trop faibles pour compromettre le déroulement ultérieur de l'hydrolyse.

Les résultats obtenus in vivo, ne permettent pas de tirer de conclusions rigoureuses sur l'hydrolyse de chacune des huiles dans la lumière du tractus gastro-intestinal. En fait, cette dernière est à la fois un lieu d'hydrolyse et d'absorption, ainsi que de remaniements des glycérides et notre type d'expérimentation ne permet d'évaluer que la résultante de tous ces phénomènes à un instant donné. On constate néanmoins que l'hydrolyse stomacale des huiles de colza, de canbra et d'arachide n'est pas négligeable, le ou les facteurs responsables de ce phénomène n'étant pas connus. D'autre part dans l'intestin grêle, aussi bien après i h 30, qu'après 3 heures, la classe glycéridique prépondérante dans les 3 lots est celle des acides gras libres, et ceci est très net dans les lots colza et arachide ; dans la lumière intestinale, l'hydrolyse des triglycérides de 1'huile de colza paraît donc aussi complète que celle de l'huile d'arachide. Aussi la moins bonne utilisation digestive de l'huile de colza ne serait pas due à un défaut d'hydrolyse de ses triglycérides, mais à une mauvaise absorption de l'acide érucique sous forme libre pour des raisons qui restent à déterminer. Des observations faites par Vodovar et al. (I972) en microscopie électronique, sur la partie jéjunale de l'intestin grêle de Rat ayant ingéré de l'huile de colza, montrent une accumulation de lipides dans la cellule absorbante pouvant produire un ralentissement de l'acheminement des lipides vers le stroma des villosités. D'autres raisons de la mauvaise absorption de l'acide érucique seraient à rechercher au niveau des formes physico-chimiques sous lesquelles cet acide gras est présent au moment de l'absorption et de la perméabilité cellulaire vis-à-vis de ces formes, ainsi qu'au niveau des spécificités enzymatiques qui conditionnent le passage de tels acides gras à travers la muqueuse intestinale. 


\section{REMERCIEMENTS}

La Société Astra-Calvé nous a fourni les huiles de colza et de canbra interestérifiées et

l’ I. T. E. R. G. les huiles de colza, canbra et arachide.

\section{SUMMARY}

\section{DIGESTIBILITY OF RAPESEED, CANBRA AND PEANUT OILS AND THEIR LIPOLYSIS IN VITRO AND IN VIVO}

The digestibility of rapeseed, canbra and peanut oils is studied by comparing the pancreatic lipase hydrolysis of these oils in vitro and in vivo in the intestinal lumen of rat.

In vitro, the initial rate at which pancreatic lipase attacks rapeseed oil is lower than in canbra and peanut oils. On the other hand, hydrolysis rate of rapeseed oil at 30 minutes is higher than in the other two oils.

In vivo, rapeseed oil slows down gastric evacuation of the diet. However, during intestinal transit, it is as completely hydrolyzed by pancreatic lipase as the other oils tested. Thus, less efficient digestive utilization of rapeseed oil is not due to deficiency of triglyceride hydrolysis, but to poor absorption of free erucic acid. After $\mathrm{I} h \mathrm{~h}$ o and 3 hours of digestion, erucic acid represents 80 p. I00 and 65 p. Ioo, respectively, of the total fatty acids found in the "free fatty acid" fraction of the small intestine.

\section{RÉFÉRENCES BIBLIOGRAPHIQUES}

Beare J. L., Murray 'T. K., Campbell J. A., i960. Response of two strains of rats to rapeseed oil and corn oil. Canad. J. Biochem. Physiol., 38, I87-192.

Bottino N. R., Vandenburg G. A., Reiser R., I967. Resistance of marine fatty acids to pancreatic lipase hydrolysis. Lipids, 2, 489-493.

Brown J. L., Johnston J. M., I962. Radioassay of lipid components separated by thin layer chromatography. I. Lipid Res., 4, 480-48I.

Clark S. B., Brause B., Holt P. R., 1969. Lipolysis and absorption of fat in the rat stomach. Gastroenterology, 56, 214-222.

Deuel H. J. (Jr.), Cheng A. L. S., Morehouse M. G., I948. The digestibility of rapeseed oil in the Rat. J. Nutrit,, 35, 295-300.

Folch J., Lees M., Sloane Stanley G. H., I957. A simple method of the isolation and purification of total lipids from animal tissues. J. Biol. Chem., 226, 497-509.

Grynberg H., Ceglowska H., Szczepanska H., ig66. Composition des triglycérides de l'huile de colza. Rev. fr. Corps gras, 10, 595-602.

Hamosh M., Scow R. O., I973. Lingual lipase and its role in the digestion of dietary lipids. J. Clin. Invest., 52, 88-95.

Marcinkiewicz J., Niewiadomski M., ig68. Modification de la structure glycéridique de l'huile de colza par suite de la réaction de transestérification. Rev. fr. Corps gras, 8-9, 5II-5 I5.

Matison F. H., BECK L.W., 1956. The specificity of pancreatic lipase for the primary hydroxyl group of glycerides. J. Biol. Chem., 219, 735-740.

Munsch N., 1970. Influence de l'ingestion de triélaidine sur la composition en acides gras des lipides hépatiques et plasmatiques de rats. Ann. Nutrit. Alim., 24, 2 I-40.

Rocouelin G., Leclerc J., i969. L'huile de colza riche en acide érucique et l'huile de colza sans acide érucique. II. Utilisation digestive comparée chez le Rat. Arn. Biol. anim., Bioch. Biophys., 9, 4I 3-426.

Rocquelin G., Sergiel J. P., Martin B., Leclerc J., Cluzan R., i97i. The nutritive value of refined rapeseed oils. A review. J. Amer. Oil Chemists'Soc., 48, 728-732. 
Savary P., Constantin M. J., rg66. Sur la résorption intestinale des chaines éruciques et leur incorporation dans les chylomicrons lymphatiques du Rat. Biochim. Biophys. Acta, 125, i 18-128.

Savary P., Desnuelle P., r956. Sur quelques éléments de spécificité pendant l'hydrolyse enzymatique des triglycérides. Biochim. Biophys. Acta, 21, 349-360.

Sergiel J. P., I973. Structure glycéridique de l'huile de canbra. Rev. fr. Corps gras, 3, I37-I4I.

SтанI, E., 1956. Dümschicht-Chromatographie. Pharmazie, 11, 633-637.

THomasson H. J., 1956. The biological value of oils and fats. IV. The rate of intestinal absorption. J. Nutrit., 59, 343-352.

Toullec R., Flanzy J., Rigaud J., ig68. Dosage des lipides des fèces. Extraction séparée, importance et composition en acides gras des lipides non saponifiés et de ceux des complexes insolubles. Ann. Biol. anim. Bioch. Biophys., 8, $28 \mathrm{I}-28 \mathrm{~g}$.

Vodovar N., Desnoyers F., Flanzy J., 1972. Absorption intestinale de l'huile de colza. Aspect ultra structural. C. R, Acad. Sc., Paris. D, 274, I743-1745.

Zrombsкi H., r964. Nutritive value of fats and their role in the development of some diseases. II. Effect of type of fat in the diet and amount of physical effort on growth of rats. Rocz. Panstwowego Zakl. Hig., 15, 52I-540. 